\title{
Message from ESSS 2013 Workshop Co-chairs
}

\author{
Jun Pang \\ University of Luxembourg \\ Luxembourg
}

\author{
Yang Liu \\ Nanyang Technological University \\ Singapore
}

\author{
Sjouke Mauw \\ University of Luxembourg \\ Luxembourg
}

The International Workshop on Engineering Safety and Security Systems (ESSS 2013) aims at contributing to the challenge of constructing reliable and secure systems. The workshop covers areas such as formal specification, (extended) type checking, model checking, program analysis/transformation, model-based testing and model-driven software construction. The workshop brings together researchers and industry R\&D expertise to exchange their knowledge, discuss their research findings, and explore potential collaborations. The first edition of ESSS was held in Feb 2012 in Singapore, organised by National University of Singapore. The ESSS 2013 workshop is affiliated with the 6th IEEE Conference on Software Testing, Verification and Validation (ICST 2013) in Luxembourg.

The main theme of the workshop is methods and techniques for constructing large reliable and secure systems. The goal of the workshop is to establish a platform for the exchange of ideas, discussion, cross-fertilisation, inspiration, co-operation, and dissemination. The topics of the workshop include, but are not limited to:

- methods, techniques and tools for system safety and security

- methods, techniques and tools for analysis, certification, and debugging of complex safety and security systems

- model-based and verification-based testing

- emerging application domains such as cloud computing and cyber-physical systems

- case studies and experience reports on the use of formal methods for analysing safety and security systems

We have received a number of 12 submissions this year. Each submission received at least 3 review reports provided by our international Program Committee (PC) and/or external reviewers. The submissions were also discussed in the virtual meeting of the PC and in the end the PC decided to select five papers (four regular papers and one short paper) among the submissions for presentation in the workshop and publication in the IEEE Digital Library in the form of a post-proceedings. In addition to the accepted presentations, we invited two internationally renowned researchers to give an invited talk. The complete workshop program provided a variety of research topics which are of current interest, including methods, techniques and tools to model-based testing, cryptographic verification of programs, code transformation and generation, compliance verification, software-based remote attestation, formal semantics of programs, and formal description of industrial standards.
We would like to express our sincere thanks and appreciation to the exceptional work rendered by the program committee members and the external reviewers for providing review reports. We want to thank all the authors for submitting their papers to the workshop, and the participants for attending it. We also would like to thank the program chairs and the organisers of ICST who provided the infrastructure for this event. Their combined efforts have made ESSS 2013 a success.

\section{Program Committee}

- Étienne André, University Paris 13, France

- Cyrille Artho, Japan Advanced Institute of Science and Technology, Japan

- Marieke Huisman, University of Twente, The Netherlands

- Weiqiang Kong, Kyushu University, Japan

- Keqin Li, SAP Research, France

- Yang Liu (PC co-chair), Nanyang Technological University, Singapore

- Zhiming Liu, UNU/IIST, China

- Sjouke Mauw (general chair), University of Luxembourg, Luxembourg

- Jun Pang (PC co-chair), University of Luxembourg, Luxembourg

- Geguang Pu, East China Normal University, China

- Mohammad Torabi Dashti, ETH Zurich, Switzerland

- Anton Wijs, Eindhoven University of Technology, The Netherlands

- Yoriyuki Yamagata, Japan Advanced Institute of Science and Technology, Japan

EXTERNAL REVIEWERS

Armin Biere, Nafees Qamar, Srdjan Marinovic 